\title{
Lysophosphatidic Acid-Induced TWIST1 and Slug Expression in Oral Cancer Cell Invasion
}

\author{
Kyung Hwa Cho ${ }^{\dagger}$ \\ Department of Pharmacology, Konyang University College of Medicine, Daejeon 35365, Korea
}

\begin{abstract}
Relative to its incidence, oral cancer has serious negative social effects. The exact causes of oral cancer have not been clarified, but many studies have implicated smoking and drinking. However, the fundamental mechanism of oral cancer causation has yet to be elucidated. Lysophosphatidic acid (LPA) augments epithelial mesenchymal transition (EMT) and development of various cancer cells. However, a detailed mechanistic explanation for LPA-induced EMT and the effects of EMT-promoting conditions on oral squamous cell carcinoma development remain elusive. In the present study, a quantitative reverse transcription polymerase chain reaction was used to analyze TWIST1, Slug, E-cadherin, and glyceraldehyde-3-phosphate dehydrogenase (GAPDH) transcript expression. Immunoblotting was used to analyze TWIST1, Slug, E-cadherin, and GAPDH protein expression. siRNAs were used to silence TWIST1 and Slug transcript expression. A matrigel-coated in vitro invasion insert was used to analyze oral cancer cell invasion. The results of the present study show that the expression levels of TWIST1 and Slug, which are EMT factors, were increased by LPA treatment in YD-10B oral squamous cell carcinoma. Conversely, E-cadherin expression was significantly reduced. In addition, transfection of the cells with TWIST1 and Slug siRNA strongly inhibited LPA-induced oral cancer cell invasion. The present study shows that TWIST1 and Slug mediate LPA-induced oral cancer cell EMT and invasiveness. The present study confirmed the mechanism by which LPA promotes oral cancer cell development, with TWIST1 and Slug providing novel biomarkers and promising therapeutic targets for oral cancer cell development.
\end{abstract}

Key Words: Epithelial-mesenchymal transition, Lysophospholipids, Mouth neoplasms

\section{Introduction}

Although oral cancer accounts for less than $5 \%$ of all cancers, it is a fatal disease with a reported 5-year survival rate of less than $60 \%$. Oral cancer has a very high recurrence rate and frequently metastasizes to lymph nodes in the neck ${ }^{1-3)}$. Metastasis of oral cancer occurs when cells become dissociated from tissue and are transported by the lymphatic and vascular systems. The cells subsequently leak out of the blood vessels and infiltrate into the tissue at the site of metastasis, where they proliferate. In terms of its prognosis, oral cancer is more closely related to postoperative social life than other cancers, and raises serious issues such as severe damage to the appearance of the face, functional impairment of chewing and speech, social affective disorders, psychological and mental health issues, and enormous treatment costs for reconstructive surgery. In other words, oral cancer results in serious impairment of quality of life. In the United States, oral cancer is reported to be due to smoking in over $77 \%$ of cases ${ }^{4)}$, while other possible causes include chronic stimulation due to poorly-fitting prostheses, syphilis and other infections, ultraviolet light, and radiation exposure. A study by Han et al. ${ }^{5)}$ in South Korea listed smoking, alcohol, and nutritional deficiency due to extreme dieting as potential causes of oral cancer.

Treatment approaches for oral cancer broadly involve chemotherapy with anticancer agents, radiotherapy, and surgery. Of these, surgical treatment is the most certain method of removing the cancer at the primary site, but it 
has limitations when the cancer has already metastasized to other organs, and additional radiotherapy and chemotherapy are often required in these cases ${ }^{5)}$.

Epithelial mesenchymal transition (EMT) refers to the process of epithelial cells changing to resemble mesenchymal cells due to a loss of cell-to-cell adhesion and cell polarity. The transition to mesenchymal cells is accompanied by an increase in the expression of EMT marker such as TWIST, Snail1/2, and vimentin. Conversely, expression of E-cadherin decreases ${ }^{6-8)}$.

The glycerophospholipid lysophosphatidic acid (LPA) is present in saliva, and becomes active in the tumor microenvironment ${ }^{9)}$. The effects of LPA are mediated by several LPA receptors, which belong to the G protein-couple receptor class ${ }^{10,11)}$. LPA promotes cancer cell migration, proliferation, and survival ${ }^{10,12,13)}$. However, the precise mechanisms by which LPA affects oral cancer metastasis have not yet been identified.

The present study aimed to investigate the effects of LPA on oral cancer cell invasion, and to suggest new therapeutic targets and biomarkers for oral cancer cell progression.

\section{Materials and Methods}

\section{Cell culture}

YD-10B oral squamous carcinoma cells were provided by Dr. J.I. Yook (Yonsei University College of Dentistry, Korea) ${ }^{14)}$, and cultured in an incubator at $5 \% \mathrm{CO}_{2}$ and $37^{\circ} \mathrm{C}$, on T75 flasks using RPMI 1640 medium containing $5 \%$ fetal bovine serum (Hyclone, Logan, UT, USA) and $1 \%$ penicillin-streptomycin (Lonza, Allendale, NJ, USA). When the cells were $80 \%$ confluent, the plates were washed twice with phosphate buffered saline solution, and the cells were subcultured using trypsin-ethylenediaminetetraacetic acid (Lonza).

\section{In vitro invasion assay}

We obtained siRNA for TWIST1 and Slug from Sigma-Aldrich (St. Louis, MO, USA). Each siRNA was transfected in accordance with the manufacturer's guidelines, using Lipofectamine RNAiMAX (Invitrogen, Carlsbad, CA, USA).
Using the method described in a previous study by Cho et al. ${ }^{15)}$, in vitro invasion was tested using a Boyden chamber (Neuro Pro Inc., Gaithersburg, MD, USA) with a membrane coated in Matrigel (BD Biosciences, San Jose, CA, USA). Addition of $1 \times 10^{5}$ cells/well into the upper chamber, with or without a pharmacological inhibitor, was performed. The lower section was filled with conditioned serum-free medium with or without LPA. After culturing for 24 hours at $37^{\circ} \mathrm{C}$, the membrane was fixed and stained using Diff-Quik (Dade Behring, Inc., Newark, DE, USA). To assess the extent of invasion, a light microscope was used to count cells in 5 randomly-selected high-magnification $(\times 200)$ fields.

\section{Quantitative reverse transcription polymerase chain reaction}

We acquired cDNA through reverse transcription using oligo (dT) primers and M-MLV reverse transcriptase (Promega, Madison, WI, USA). For this purpose, we used total RNA that had been isolated using Tri-reagent (Ambio, Austin, TX, USA). The cDNA was amplified using a CFX96 reverse transcription polymerase chain reaction (RT-PCR) detection system (Bio-Rad Life Sciences, Hercules, CA, USA). The reaction mixture for quantitative RT-PCR included SYBR Green PCR master mix (Bio-Rad Life Sciences), template cDNA, the individual primers, TWIST1, Slug, E-cadherin, and glyceraldehyde-3-phosphate dehydrogenase (GAPDH). The total volume of the mixture used was $20 \mu \mathrm{l}$. PCR amplification was conducted using the following specific primer sets: TWIST1: forward 5'-GTC CGC AGT CTT ACG AGG AG-3'/reverse 5'-CCA GCT TGA GGG TCT GAA TC-3'; Slug: forward 5'-TCT GCA GAC CCA TTC TGA TG-3'/5'-AGC AGC CAG ATT CCT CAT GT-3'; E-cadherin: forward 5'-ACA GCC CCG CCT TAT GAT T-3'/5'-TCG GAA CCG CTT CCT TCA-3'; GAPDH: forward 5'-ACA GTC AGC CGC ATC TTC TT-3' reverse, 5'-ACG ACC AAA TCC GTT GAC TC-3'. GAPDH was used as a control for calculating dCt. The RT-PCR data were analyzed using the 2-(ddCt) method.

\section{Immunoblotting}

The cell solution was prepared as described in the study 
by Cho et al. ${ }^{15)}$. TWIST1 and GAPDH antibodies were obtained from Santa Cruz Biotechnology Inc. (Santa Cruz, CA, USA). Slug antibody was obtained from Cell Signaling Technology (Danvers, MA, USA). E-cadherin antibody was obtained from BD Biosciences. The immunoreactive bands were visualized by ECL (Pierce, Rockford, IL, USA) using an ImageQuant 300 (GE Healthcare, Buckinghamshire, UK).

\section{Statistical analysis}

All data were analyzed using IBM SPSS Statistics ver. 22.0 (IBM Co., Armonk, NY, USA).
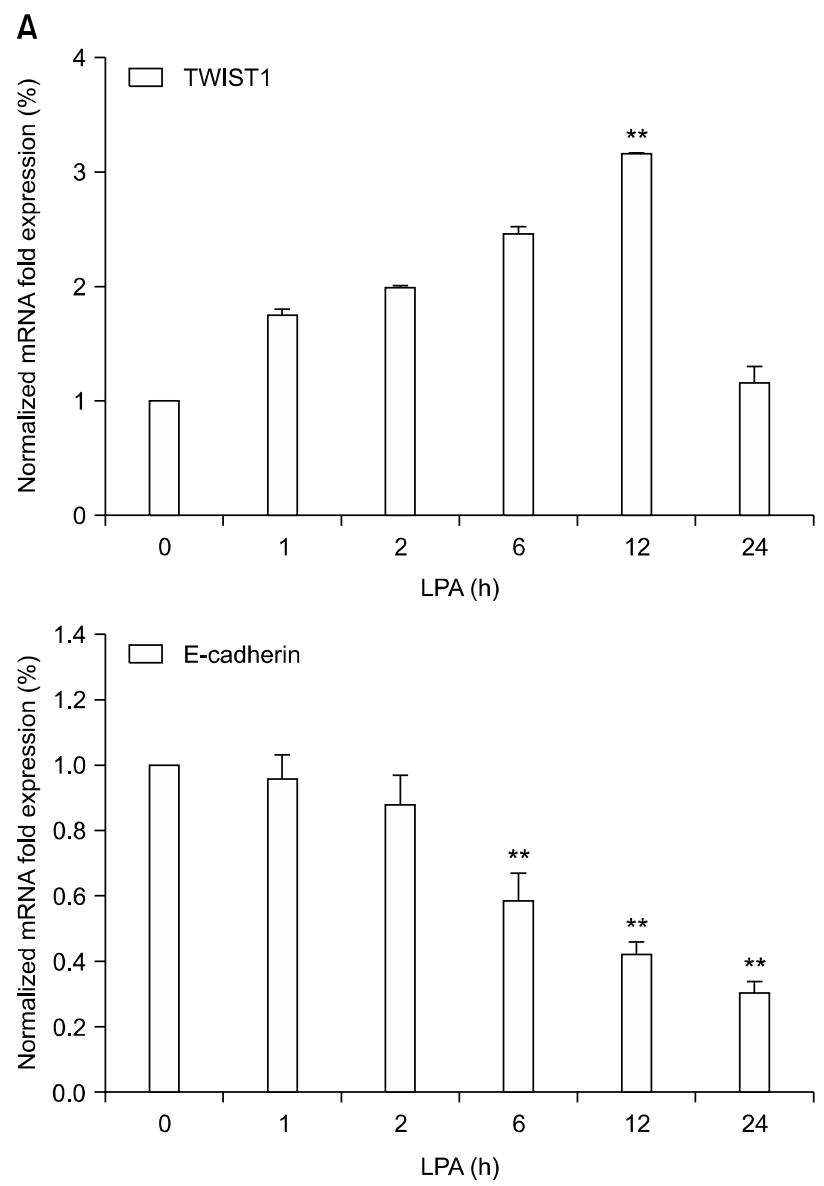

\section{Results}

\section{Effects of LPA on expression of EMT factors}

In order to investigate the effects of LPA on expression of EMT factors, we treated YD-10B cells with LPA at different times, and measured the mRNA (Fig. 1A) and protein (Fig. 1B) expression of TWIST1, Slug, and E-cadherin, which are known to be important factors in EMT. We found that mRNA expression was the highest for TWIST1 at 12 hours and for Slug at 2 hours (Fig. 1A). Protein expression was the highest for TWIST1 at 24 hours and for Slug at 6 hours (Fig. 1B). Conversely, for E-cadherin, both mRNA and protein expression decreased with increasing time (Fig. 1). These results demonstrate that LPA promotes EMT in oral cancer cells.

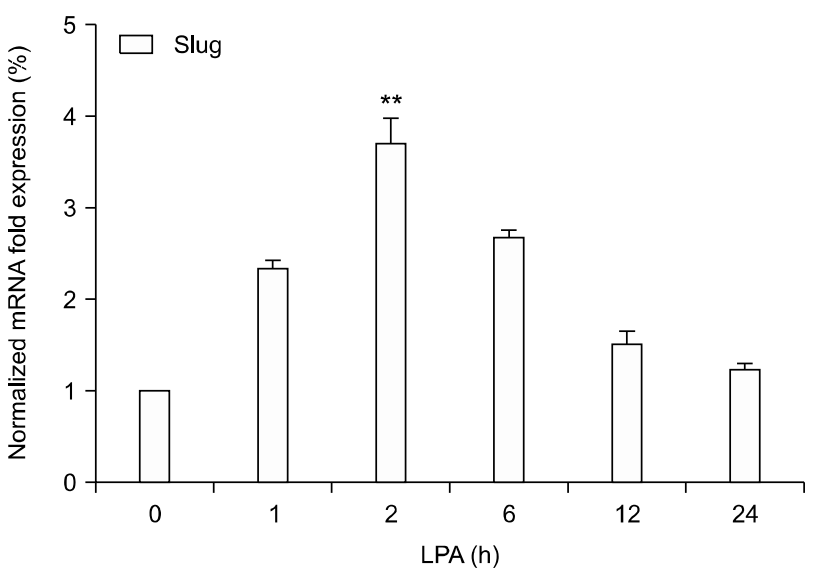

B

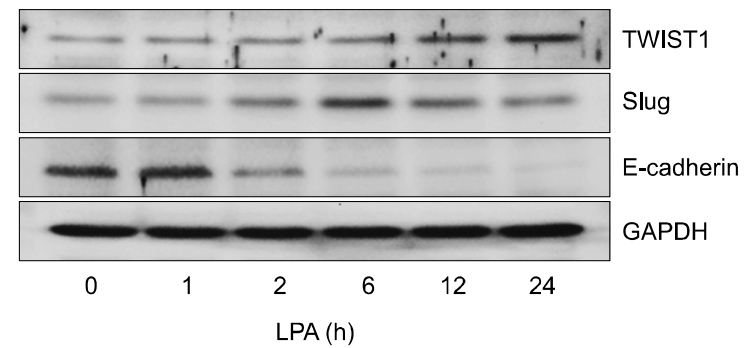

Fig. 1. Lysophosphatidic acid (LPA) induces epithelial mesenchymal transition factors in oral cancer cells. The YD-10B cells were serum-starved and stimulated with LPA $(10 \mu \mathrm{M})$ for indicated times. Quantitative reverse transcription polymerase chain reaction (A) and immunoblotting (B) (error bars, mean \pm standard deviation; $\star * p<0.01$ vs. control). All experiments were repeated three times. GAPDH: glyceraldehyde-3-phosphate dehydrogenase. 


\section{The importance of TWIST1 and Slug in LPA-induced oral cancer cell invasion}

We performed a Boyden chamber assay to verify whether LPA caused an increase in invasion by oral cancer cells. As shown in Fig. 2, we found that YD-10B cells showed a considerable increase in invasion when treated with LPA. However, TWIST1 and Slug siRNA silenced TWIST1 expression, resulting in significant inhibition of LPA-induced oral squamous carcinoma cell invasion. These results demonstrate that TWIST1 and Slug regulate the invasion of oral squamous carcinoma cell induced by LPA.

\section{Discussion}

Relative to its incidence, oral cancer has a severe negative effect on social life. Although the precise causes of oral cancer have not been identified, a number of studies have reported smoking and alcohol to be important factors ${ }^{4,16)}$. Nevertheless, since the basic mechanisms of oral cancer have still not been clarified, we aimed to investigate these mechanisms.

LPA is a phospholipid that is known to produce various cellular responses via stimulation of $\mathrm{G}$ protein families such as $\mathrm{Gi}, \mathrm{G} 12$, and $\mathrm{Gq}^{17-19)}$. Subsequent to the finding of high expression in ovarian cancer patients, LPA has been reported to be involved in processes in various cells that have an important effect on the development of cancer, such as proliferation, migration, and survival ${ }^{20)}$. In animal models, LPA has been reported to be associated with breast cancer metastasis ${ }^{21)}$.

TWIST1 is a highly-conserved, basic helix-loop-helix
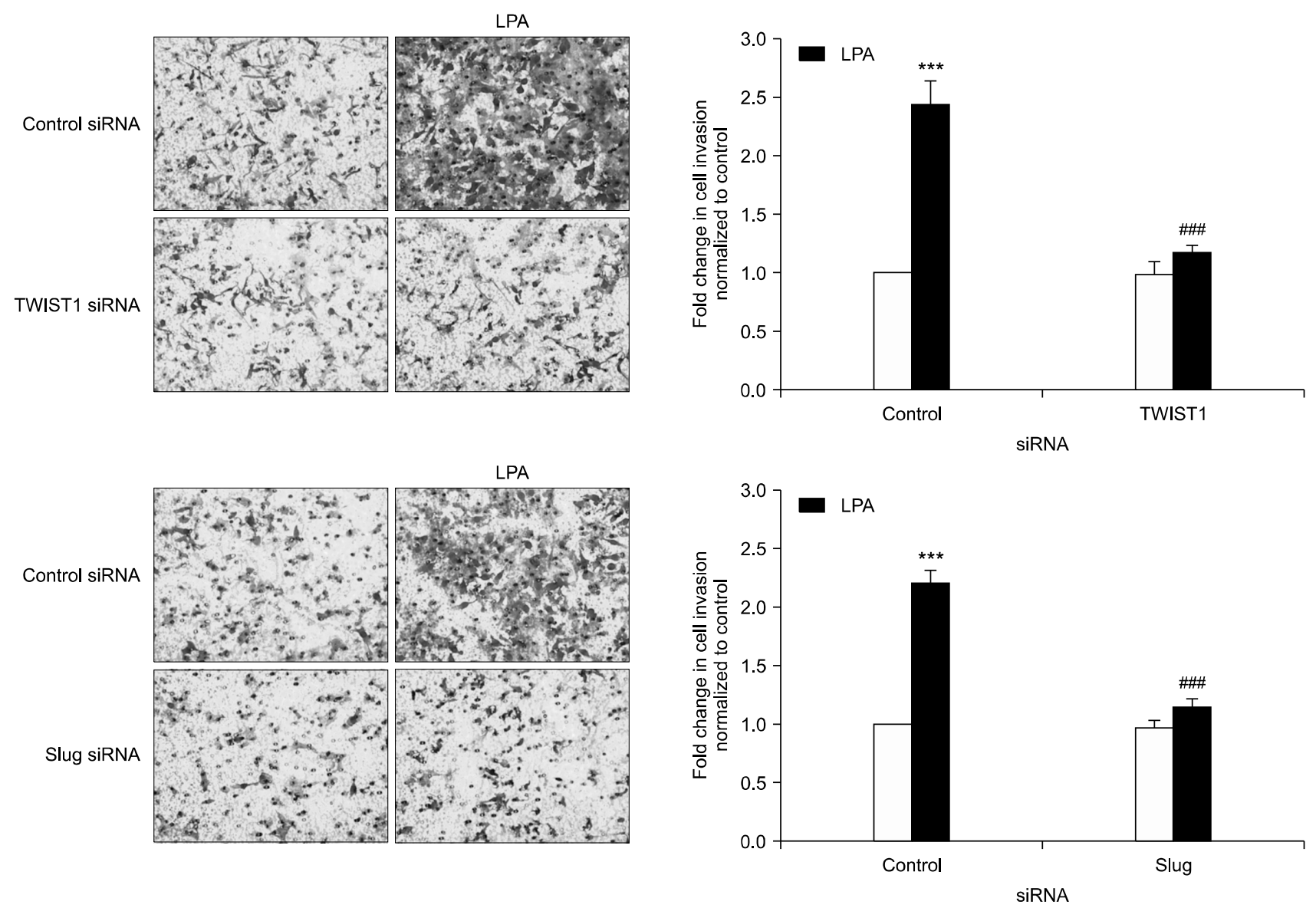

Fig. 2. Epithelial mesenchymal transition factors is important for lysophosphatidic acid (LPA)-induced oral cancer cell invasion. The YD-10B cells were transfected with indicated siRNAs, and in vitro invasion were analyzed against LPA (10 $\mu M$ ) (error bars, mean \pm standard deviation; $* * * \mathrm{p}<0.001$ vs. scrambled control siRNA, $\# \#$ $p<0.001$ vs. scrambled control siRNA and LPA treatment). All experiments were repeated three times. 
transcription factor, and acts as a major regulator of gastrulation and mesoderm specification ${ }^{22)}$. Slug is a zinc finger transcription factor, also known as Snail2, which is very closely related to embryonic development and cancer metastasis. TWIST1 and Slug prevent oncogene-mediated cell aging and cell death, and increase resistance to chemotherapy. Moreover, these factors strengthen cancer stem-cell populations, and promote invasion and metastasis of cancer cells ${ }^{23,24)}$.

Recent studies have reported a molecular mechanism by which TWIST1 and Slug promote EMT and cancer cell migration, invasion, and metastasis by inhibiting E-cadherin expression ${ }^{23,24)}$. Fundamentally, the polarized epithelial cells found in organized single or stratified cell layers change into motile, single-layer fibroblasts. Essential cellular changes include loss of epithelial polarity, epithelial cell-cell adhesion and cell--matrix interaction and reorganization of the actin cytoskeleton. Motile fibroblasts activate a molecular program that is capable of widespread dissolution and neosynthesis of the extracellular matrix (ECM). The nature of the reorganized matrix allows the transformed motile cells to pass through the basement membrane and continue migrating through the ECM, a process that defines in particular tumor invasiveness. Thus, these distinct but interrelated cellular changes are referred to as phenotypic modules, and can be considered a definition for the complete EMT phenotype of nonmalignant and malignant cells subjected to morphological pressure ${ }^{25)}$.

In the present study, we verified LPA-induced EMT and invasion effects in oral cancer cells. Our results demonstrate not only that LPA causes an increase in expression of 2 important EMT factors, TWIST1 and Slug in oral cancer, but also that silencing TWIST1 and Slug caused a significant decrease in LPA-induced invasion by oral cancer cells. This means that TWIST1 and Slug are important biomarkers for LPA-induced oral cancer cell invasion.

Our study is important because it investigates the effects of LPA on oral cancer cell invasion. However, because the experiments were conducted at the cell level, additional animal experiments and clinical studies will be required. If a drug were developed that could suppress mechanisms of
LPA-induced oral cancer cell metastasis, we expect it would show a revolutionary inhibitory effect on oral cancer. Accordingly, active drug research and development are needed.

\section{Acknowledgements}

This research was supported by a grant from Basic Science Research Program through the National Research Foundation of Korea (NRF) funded by the Ministry of Eucation, Science and Technology (No. 2017R1A2B4007631).

\section{References}

1. Roepman P, Wessels LF, Kettelarij N, et al.: An expression profile for diagnosis of lymph node metastases from primary head and neck squamous cell carcinomas. Nat Genet 37: 182-186, 2005.

2. Kim J, Park YM, Lim DS: Purification of materials produced by Amylocolatosis sp. and anticancer effect in oral cancer model. J Dent Hyg Sci 3: 11-14, 2003.

3. Kim JH, Jung YS, Kim HY, et al.: The anti-cancer effect of $\beta$-gluconsan calcium on oral cancer cell. J Dent Hyg Sci 15: 794-799, 2015.

4. Rodriguez T, Altieri A, Chatenoud L, et al.: Risk factors for oral and pharyngeal cancer in young adults. Oral Oncol 40: 207-213, 2004.

5. Han JH, Kim EK, Lim SH, Kim CH: Literature review on the incidence and risk factor of oral cancer. J Dent Hyg Sci 12: 451-458, 2012.

6. Nieto MA: The snail superfamily of zinc-finger transcription factors. Nat Rev Mol Cell Biol 3: 155-166, 2002.

7. Beavon IR: The E-cadherin-catenin complex in tumour metastasis: structure, function and regulation. Eur J Cancer 36: 1607-1620, 2000.

8. Yang J, Weinberg RA: Epithelial-mesenchymal transition: at the crossroads of development and tumor metastasis. Dev Cell 14: 818-829, 2008.

9. Bathena SP, Huang J, Nunn ME, et al.: Quantitative determination of lysophosphatidic acids (LPAs) in human saliva and gingival crevicular fluid (GCF) by LC-MS/MS. J Pharm Biomed Anal 56: 402-407, 2011. 
10. Houben AJ, Moolenaar WH: Autotaxin and LPA receptor signaling in cancer. Cancer Metastasis Rev 30: 557-565, 2011.

11. Anliker B, Chun J: Cell surface receptors in lysophospholipid signaling. Semin Cell Dev Biol 15: 457-465, 2004.

12. Mills GB, Moolenaar WH: The emerging role of lysophosphatidic acid in cancer. Nat Rev Cancer 3: 582-591, 2003.

13. Choi JW, Herr DR, Noguchi K, et al.: LPA receptors: subtypes and biological actions. Annu Rev Pharmacol Toxicol 50: 157-186, 2010.

14. Nam W, Tak J, Ryu JK, et al.: Effects of artemisinin and its derivatives on growth inhibition and apoptosis of oral cancer cells. Head Neck 29: 335-340, 2007.

15. Cho KH, Jeong KJ, Shin SC, Kang J, Park CG, Lee HY: STAT3 mediates TGF- $\beta 1$-induced TWIST1 expression and prostate cancer invasion. Cancer Lett 336: 167-173, 2013.

16. Warnakulasuriya S, Parkkila S, Nagao T, et al.: Demonstration of ethanol-induced protein adducts in oral leukoplakia (pre-cancer) and cancer. J Oral Pathol Med 37: 157-165, 2008.

17. Bian D, Mahanivong C, Yu J, et al.: The G12/13-RhoA signaling pathway contributes to efficient lysophosphatidic acid-stimulated cell migration. Oncogene 25: 2234-2244, 2006.
18. Bian D, Su S, Mahanivong C, et al.: Lysophosphatidic acid stimulates ovarian cancer cell migration via a Ras-MEK kinase 1 pathway. Cancer Res 64: 4209-4217, 2004.

19. Moolenaar WH, van Meeteren LA, Giepmans BN: The ins and outs of lysophosphatidic acid signaling. Bioessays 26 : 870-881, 2004.

20. Moolenaar WH: Development of our current understanding of bioactive lysophospholipids. Ann N Y Acad Sci 905: 1-10, 2000.

21. Boucharaba A, Serre CM, Grès S, et al.: Platelet-derived lysophosphatidic acid supports the progression of osteolytic bone metastases in breast cancer. J Clin Invest 114: 1714-1725, 2004.

22. Thisse B, el Messal M, Perrin-Schmitt F: The twist gene: isolation of a Drosophila zygotic gene necessary for the establishment of dorsoventral pattern. Nucleic Acids Res 15: 3439-3453, 1987.

23. Qin Q, Xu Y, He T, Qin C, Xu J: Normal and disease-related biological functions of Twist 1 and underlying molecular mechanisms. Cell Res 22: 90-106, 2012.

24. Shih JY, Yang PC: The EMT regulator slug and lung carcinogenesis. Carcinogenesis 32: 1299-1304, 2011.

25. Zavadil J, Böttinger EP: TGF-beta and epithelial-to-mesenchymal transitions. Oncogene 24: 5764-5774, 2005. 